\title{
A MULTIPLE-MODEL APPROACH FOR SYNCHRONOUS GENERATOR NONLINEAR SYSTEM IDENTIFICATION
}

\author{
Seyed Salman Ahmadi — Mehdi Karrari *
}

\begin{abstract}
In this paper, a multiple model approach is proposed for the identification of synchronous generators. In the literature, the same structure often is used for all local models. Therefore, to obtain a precise model for the operating condition of the synchronous generator with severely nonlinear behavior, many local models are required. The proposed method determines the complexity of local models based on complexity of behavior of the synchronous generator at different operating conditions. There are two choices for increasing model precision at each iteration of the proposed method: (i) increasing the number of local models in one region, or (ii) increasing local model complexity in the same region. The proposed method has been tested on experimental data collected on a $3 \mathrm{kVA}$ micro-machine. In the study, the field voltage is considered as the input and the active output power and the terminal voltage are considered as the outputs of the synchronous generator. The proposed method provides a more precise model with fewer parameters compared to some well known methods such as LOLIMOT and global polynomial models.
\end{abstract}

K e y w or ds: multiple model approach, power system identification, power system modeling, synchronous generator

\section{INTRODUCTION}

With the increased complexity of the interconnected power systems, modeling of such systems has become critical. These systems contain a large number of synchronous generators. An important step in modeling of the interconnected power systems is to obtain a valid model of synchronous generator. Synchronous generator is a nonlinear system. If some practical nonlinearities, such as the magnetic saturation of the stator and rotor iron are considered, the system shows severely nonlinear properties.

Synchronous generator modeling can be classified into two approaches. The first approach [1-5] assumes a known structure for the synchronous generator and the physical parameters are estimated. For example, a stepby-step procedure to estimate the parameter values of the $d-q$ axis synchronous machine models using the standstill Frequency response testing is presented in [1]. In [2], a closed loop subspace parameter identification technique is proposed to estimate Heffron-Phillips model parameters. Such a technique is used instead of open-loop identification to avoid bias errors in the estimated parameters.

In the second approach (synchronous generator identification) [6-8], no physical insight is used, but the structure belongs to families that are known to have good flexibility. The structure parameters are estimated from input-output measurements. Such a model is used either in a predictive control structure for applications such as on-line power system stabilizer design, or used as a simulator to test an off-line design.

Many different structures, like Volterra, Hammerstein series [9], Hammerstein-Wiener [10], wavelet network and neural network have been presented in the literature. In order to model the behavior of a synchronous generator at different operating conditions, these structures require a huge number of parameters. Several methods such as orthogonal projection pursuit [11], relative contribution [12] and orthogonal least square with A-optimality design method [13] have been developed for selecting the most significant terms of such structures.

Another approach for modeling of synchronous generator is multiple model approach $[14,15]$. In such an approach, each local model shows the behavior in a region of the synchronous generators operating space. Therefore, to obtain a precise model for the operating region with severely non-linear behavior, many local models are required. In multiple model methods, depending on the level of nonlinearity, the number of local models and the number of terms for each local model, many parameters need to be estimated.

In [15], multiple model approach is used to model synchronous generator. Partitioning of operating space is obtained using nonlinear optimization. After each iteration of nonlinear optimization, all local linear models parameters are estimated by global learning. Therefore, the model loses the local interpretability. In multiple model structures using global learning the behavior of local models may not change smoothly as a function of the operating region. The use of such non smooth models can lead to unreliable control.

The parameters of local models are usually estimated by global or local learning algorithm. The local learning is faster than global learning. The local learning leads to local interpretability which means that the local models

* Electrical Engineering Department, Amirkabir University of Technology, Tehran, Iran 
reflect the process behavior at the corresponding operating region, but such property is not satisfied by global learning. The number of effective parameters in model is less with local learning than with global learning (see [16] and references therein).

Therefore, the parameters are estimated by local learning algorithm in the proposed method. In this paper, an effort is made to present a new method combining the advantages of both non-linear identification methods. This new method uses the multiple model structure. Where necessary, use local polynomial model instead of local linear model. In the proposed method, global model can be obtained with fewer local models. Contrary to the abovementioned methods, in the proposed method, not only the number of local models is increased, but there are two choices for model increased precision: (1) Increase in the number of local models in one region, and (2) increase in local model complexity in the same region. The local models complexity is, therefore, different at different operating regions. In the proposed method, the iteration of algorithm may not result in a new local model but in the increased complexity of one of the local models.

In Section 2, the formulation of the multiple model approach is described. In Section 3 the proposed algorithm is presented. Experimental setup and data collection on a micro-machine are discussed in Section 4. In Section 5 the proposed method is applied on the experimental data of the micro-machine, and compared with other methods. Section 6 concludes the paper.

\section{THE MULTIPLE MODEL APPROACH}

In this approach, a global model is given by the combination of the local models, which have been identified over different operating conditions of the synchronous generator. In these methods, global output $\hat{y}(k)$ is equal to the weighted sum of $M$ local models $y_{i}(k), i=1, \ldots, M$, that is

$$
\hat{y}(k)=\sum_{i=1}^{M} y_{i}(k) \Phi_{i}(\mathbf{z}(k))
$$

with $y_{i}(k)=f_{i}\left(\boldsymbol{\varphi}_{i}(k), \boldsymbol{\theta}_{i}\right)$, where $\boldsymbol{\varphi}(\cdot)$ and $\boldsymbol{\theta}_{i}$ are the regression and parameter vectors, respectively, and $z(k)=$ $\left[z_{1}(k), \ldots, z_{1}(k)\right]^{\top}$ is the operating space vector. The validity function $\Phi_{i}(\boldsymbol{z}(k))$ describes the contribution of the $i$-th local model to the output. Usually, the validity function $\Phi_{i}(\boldsymbol{z}(k))$ is defined as

$$
\Phi_{i}(\mathbf{z}(k))=\frac{\rho_{i}\left(\mathbf{z}(k), \boldsymbol{c}_{i}, \boldsymbol{d}_{i}\right)}{\sum_{j=1}^{M} \rho_{j}\left(z(k), \boldsymbol{c}_{j}, \boldsymbol{d}_{j}\right)}
$$

where $\rho_{i}(\cdot)$ are often chosen to be multi-dimensional Gaussian functions and $\boldsymbol{c}_{i}=\left[c_{i, 1}, c_{i, 2}, \ldots, c_{i, q}\right]^{\top}$ and $\boldsymbol{d}_{i}=\left[d_{i, 1}, d_{i, 2}, \ldots, d_{i, q}\right]^{\top}$ are, respectively, the centre and the standard deviation of multi-dimensional Gaussian function. In other words, global output is obtained by the average of the local model outputs $y_{i}(k)$ weighted by local model validity function.

The local models $f_{i}(\cdot, \cdot)$ used are linear in parameters. The $\boldsymbol{\varphi}_{i}(\cdot)$ and $\boldsymbol{z}(k)$ can be chosen independently. These vectors can be composed of previous model output and input.

The partitioning strategy of the operating space determines the validity functions parameters. If no a priori knowledge can be utilized for the partitioning of the operating space, either a grid partition or a data-driven method has to be chosen. An overview of existing partitioning strategies is given in [16].

In this paper, the partitioning of operating space is not assumed to be known a priori. The proposed method determines the most significant local models terms. Also the local model orders can be determined. The local models and validity functions parameters are estimated by linear optimization.

\section{THE PROPOSED METHOD}

Assuming a set of input output data is available; first a global model is fitted to the available input-output dataset $\{y(k), u(k)\}_{k=1}^{N}$ where $y(k)$ and $u(k)$ are output and input measurements, respectively. Next, the system operating space is split into two halves along all dimensions of operating space. For each division, a local model is considered, that is,

$$
y(k)=y_{1}(k) \Phi_{1}(\mathbf{z}(k))+y_{2}(k) \Phi_{2}(\mathbf{z}(k)) .
$$

The local models used are quadratic polynomial. The parameters of the two local models are estimated such that the two models local weighted least squares are minimized together. The local weighted least squares are as follow

$J_{i}=\sum_{k=1}^{N} \Phi_{i}(\mathbf{z}(k))\left(y(k)-y_{i}(k)\right)^{2}=\left(\boldsymbol{y}-\boldsymbol{y}_{i}\right)^{\top} Q_{i}\left(\mathbf{y}-\boldsymbol{y}_{i}\right)$,

$i=1,2$

where $Q_{i}=\operatorname{diag}\left(\Phi_{i}(\boldsymbol{z}(1)), \Phi_{i}(\boldsymbol{z}(2)), \ldots, \Phi_{i}(\boldsymbol{z}(N))\right)$.

The local weighted least squares $\left(i e J_{1}, J_{2}\right)$ are combined to one

$$
\begin{gathered}
J=J_{1}+J_{2}=\left(L_{1} \boldsymbol{y}-L_{1} \mathbf{y}_{1}\right)^{\top}\left(L_{1} \boldsymbol{y}-L_{1} \boldsymbol{y}_{1}\right) \\
+\left(L_{2} \boldsymbol{y}-L_{2} \mathbf{y}_{2}\right)^{\top}\left(L_{2} \boldsymbol{y}-L_{2} \mathbf{y}_{2}\right)= \\
\left(\left[\begin{array}{l}
L_{1} \boldsymbol{y} \\
L_{2} \boldsymbol{y}
\end{array}\right]-\left[\begin{array}{cc}
L_{1} U & 0_{N \times n} \\
0_{N \times n} & L_{2} U
\end{array}\right]\right)^{\top}\left(\left[\begin{array}{c}
L_{1} \boldsymbol{y} \\
L_{2} \boldsymbol{y}
\end{array}\right]-\left[\begin{array}{cc}
L_{1} U & 0_{N \times n} \\
0_{N \times n} & L_{2} U
\end{array}\right]\right) .
\end{gathered}
$$

In equation (5), $Q_{i}, i=1,2$ is decomposed into $Q_{i}=$ $L_{i}^{\top} L_{i}$ where $L_{i}$ is an upper triangular matrix with positive diagonal elements and $\boldsymbol{y}_{i}=U \boldsymbol{\theta}_{i}$, the memory matrix $U$ is composed of candidate regressors. 


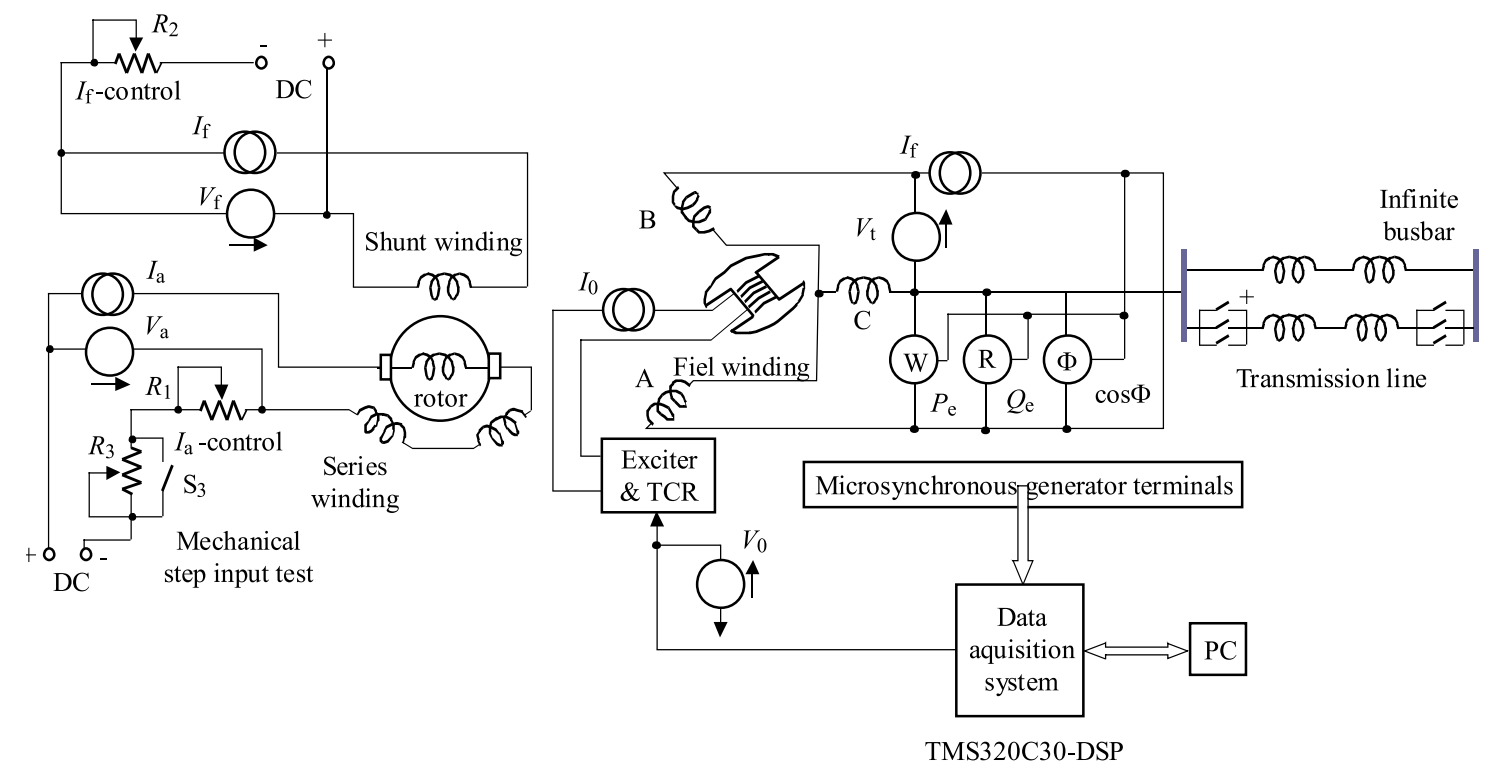

Fig. 1. Experimental setup for the micro-machine

Now an orthogonal least squares with A-optimality algorithm is used in order to determine the most significant terms and to estimate the local models parameters, $y_{1}(k)$ and $y_{2}(k)$. See the Appendix for more details of the Orthogonal Least Squares with A-optimality algorithm.

If all chosen terms correspond only to one of the two local models (for example, $y_{1}(k)$ ), the other model along with the corresponding membership function can be eliminated. In such a case it is recommended the orthogonal least squares with A-optimality method be implemented once more to determine the most significant terms of $y_{\text {new }}(k)=y_{1}(k)$ ie

$$
\begin{aligned}
J=J_{1}=\left(\boldsymbol{y}-\boldsymbol{y}_{1}\right)^{\top} Q & \left(\boldsymbol{y}-\boldsymbol{y}_{1}\right)= \\
& \left(L \boldsymbol{y}-L U \boldsymbol{\theta}_{1}\right)^{\top}\left(L \boldsymbol{y}-L U \boldsymbol{\theta}_{1}\right)
\end{aligned}
$$

where $Q$ is the validity function corresponding to the worst model. The matrix $Q$ is decomposed into $Q=$ $L^{\top} L$, where $L$ is an upper triangular matrix with positive diagonal elements.

With the above-mentioned method, new local models are obtained for all operating dimension $m=1, \ldots, q$. Among all partitions, the partition with the smallest output error is chosen. Then, the local loss functions for each local model $\left(S_{j}, j=1, \ldots, n l\right.$ where $n l$ denotes the number of local models) are computed by weighting the squared model errors with the corresponding value of validity function

$$
S_{j}=\sum_{k=1}^{N} \Phi_{j}(\boldsymbol{z}(k))(y(k)-\hat{y}(k))^{2}, \quad j=1, \ldots, n l .
$$

Next the local model with the maximum local loss function is chosen. Now the algorithm is repeated with regard to the operating region of the chosen local model. The proposed algorithm is outlined below:
1. Identify a global model using the orthogonal least squares with A-optimality algorithm.

2. Calculate the local loss function in (7) for all local models. Choose the worst local model with the highest local loss function.

3. Split the rectangle of the worst local model into two halves with an axis-orthogonal split. Try divisions in all dimensions. Carry out the following steps for each division:

3a. Construct membership functions $\rho_{i}(\cdot)$ for both rectangles. The centers of these membership functions are the centers of the rectangles. The standard deviation in each dimension is calculated as $\sigma_{m}=k \Delta_{m}$, where $\Delta_{m}$ is the width of the rectangle in the dimension $m=$ $1,2, \ldots, q$ and $k$ a factor which is chosen a priori, This parameter determines a smooth transition between local models.

3b. Construct all validity functions.

3c. Using (5) and the orthogonal least squares with Aoptimality algorithm determine a number of $y_{1}(k)$ and $y_{2}(k)$ terms as most significant terms and estimate their parameters.

$3 \mathrm{c}-1$. If all the chosen terms are only corresponding to one of the two local models, implement the orthogonal least squares with A-optimality method for (6).

$3 d$. Calculate the sum of square errors for the global model (global loss functions).

4. Among all divisions constructed in step 3, select the one with the smallest global loss function.

5. Check the termination criterion: if satisfied, algorithm is stopped. Otherwise go to step 2.

In the proposed algorithm, the significant terms of both local models in one dimension are estimated together. In this method, it is decided which of the two selections is better to achieve a more precise model: Increasing the number of local models (if the selected parameters 

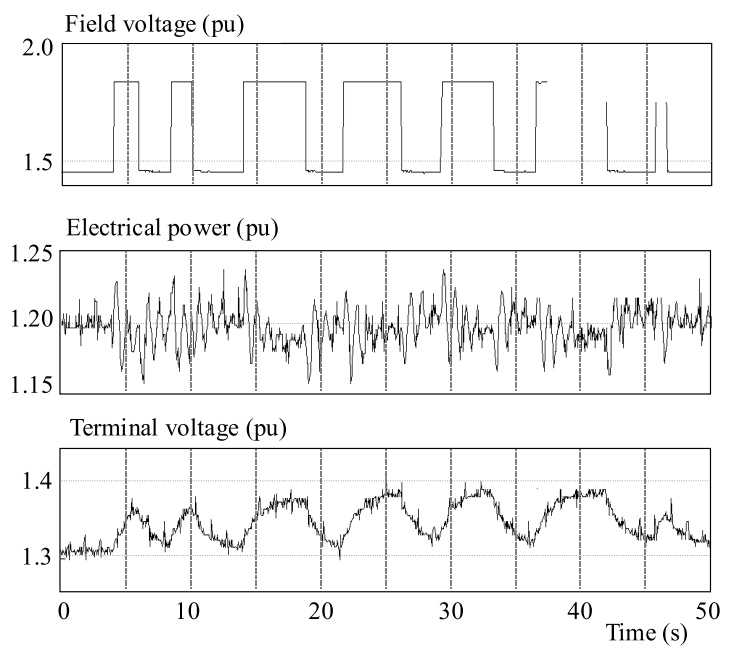

Fig. 2. Experimental data with a PRBS signal applied to the field

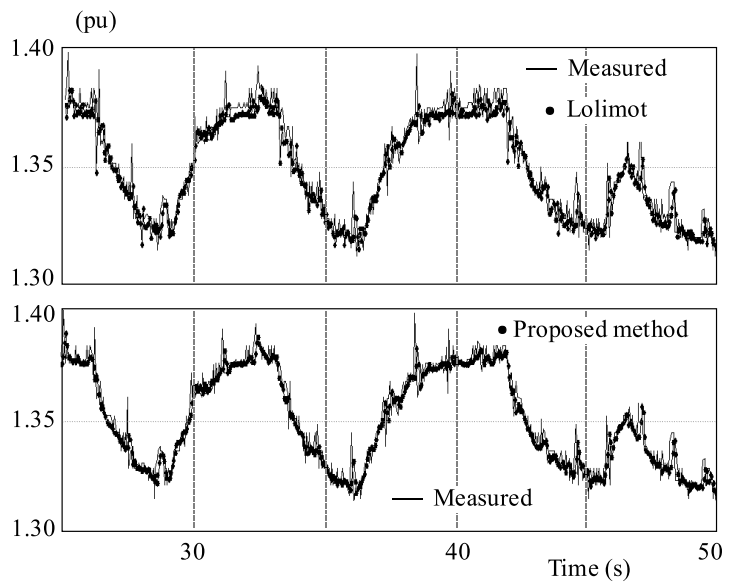

Fig. 3. Performance of LOLIMOT and the proposed algorithm for terminal voltage on the validation data
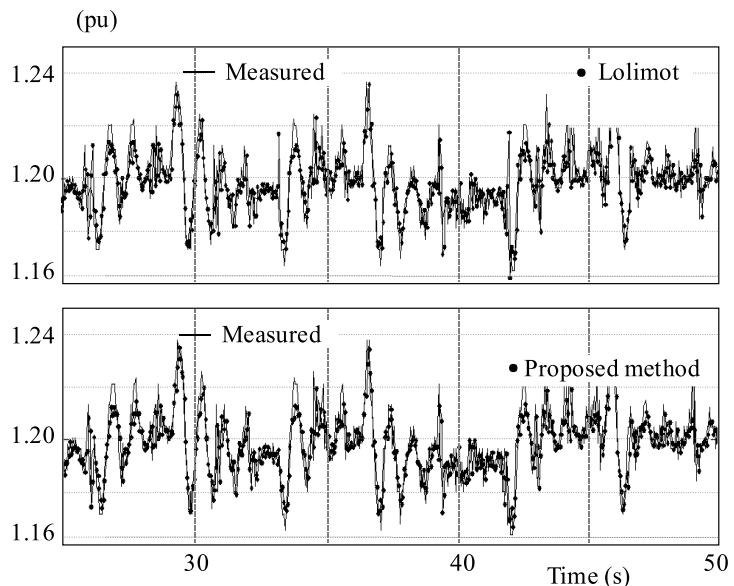

Fig. 4. Performance of LOLIMOT and the proposed algorithm for electrical power on the validation data

belong to both or regions), or increasing the number of terms (if the selected parameters belong to one of the regions). However, in LOLIMOT algorithm [16], a local linear model is identified for both partitions. The complexity of both identified models is the same. Since there is the possibility that there may not be sufficient data in one of these regions, the resulting model will not be precise. This problem is solved in the proposed method.

\section{EXPERIMENT ON SYNCHRONOUS GENERATOR}

The system under consideration is a $3 \mathrm{kVA}, 208 \mathrm{~V}, 3$ phase micro-machine, driven by a DC motor. The micro machine can represent dynamic response of much larger synchronous machines when the parameters and variables are considered in a normalized version (per unit system [17]). The main problem with a micro-machine can be the field time constant, which is much lower than that of the larger machines. This problem has been overcome using a time constant regulator, which is used to increase the effective field time constant to match that of the larger units.

The experimental setup used for the experiment is shown in Fig. 1. The synchronous generator is driven by a DC motor. The exciter input signal is applied to the synchronous machine through a D/A converter. The field voltage, terminal voltage $\left(v_{t}\right)$ and the electrical power $(P)$ are measured and sampled by the data acquisition system. The machine is connected to a constant voltage bus by a double circuit transmission line modeled by lumped elements. Each circuit consists of six $\pi$ sections and simulates the performance of a $300 \mathrm{~km}$ long $500 \mathrm{kV}$ transmission line.

The sampling time was selected to be $50 \mathrm{~ms}$. This sampling time proved to be fast enough to capture the required dynamics.

A pseudo random binary sequence (PRBS) signal with $25 \%$ of the nominal value was applied. The initial operating condition was selected to be $P=1.2 \mathrm{pu}, Q=0.72 \mathrm{pu}$, $v_{t}=1.31 \mathrm{pu}$.

The field voltage, electrical power and terminal voltage measured are shown in Fig. 2.

\section{SIMULATION RESULTS}

In this section, the number of effective parameters and the precision of the proposed algorithm for the experimental results (in Section 4), are compared with other methods.

The number of effective parameters of global quadratic model is considered to be equal to the number of global model's parameters. In local learning, the number of effective parameters is given by [16]

$$
\begin{aligned}
n=\sum_{i=1}^{n l} \sum_{j=1}^{n l} \operatorname{tr}\left(Q_{i} U_{i}\left(U_{i}^{\top} Q_{i} U_{i}\right)^{-1} U_{i}^{\top} Q_{i}\right. \\
\left.\times Q_{j} U_{j}\left(U_{j}^{\top} Q_{j} U_{j}\right)^{-1} U_{j}^{\top} Q_{j}\right)
\end{aligned}
$$

where $\operatorname{tr}(A)$ denotes the trace of a matrix $A$ and $U_{i}$, the memory matrix, corresponds to the $i$-th local model. 
Table 1. Results for terminal voltage

\begin{tabular}{ccc}
\hline model & LOLIMOT & $\begin{array}{c}\text { The proposed } \\
\text { method }\end{array}$ \\
\hline$n$ & 37 & 12 \\
Number of local models & 12 & 2 \\
NRMSE & 0.3296 & 0.2948 \\
\hline
\end{tabular}

Table 2. Results for electrical power

\begin{tabular}{ccc}
\hline model & LOLIMOT & $\begin{array}{c}\text { The proposed } \\
\text { method }\end{array}$ \\
\hline$n$ & 55 & 14 \\
Number of local models & 20 & 3 \\
NRMSE & 0.6251 & 0.5819 \\
\hline
\end{tabular}

The models are trained using the first 500 samples of the input-output data set and tested with the remaining 500 samples. The order of local models is considered to be equal three. In this study, the input is the sampled field voltage and the outputs are the sampled terminal voltage and electrical power. The operating space for LOLIMOT and the proposed algorithm is chosen as $z(k)=[u(k-1), y(k-1)]^{\top}$. The validation capability and accuracy of different methods could be evaluated using the Normalized Root Mean Square Error (NRMSE).

Now that the system under study and the criteria for comparison are explained, the proposed identification is applied on the system and compared with a global quadratic model and LOLIMOT methods. First the main design points for each approach are explained:

Global quadratic model: To select the most significant model terms and estimate the model parameters simultaneously, the orthogonal least squares with A-optimality algorithm is used. The results of LOLIMOT and the proposed algorithms are by far better than that provided by global quadratic model. Therefore, the efficiency of the proposed identification method and the LOLIMOT method is discussed.

LOLIMOT algorithm: In continuous iteration of LOLIMOT algorithm, identified models are tested.

The proposed algorithm: This method with $\alpha=10^{-6}$ is applied for electrical power and terminal voltage. The identified models are validated in various iterations.

The model output for terminal voltage and electrical power over the validation data identified by LOLIMOT and the proposed algorithms are shown in Figs. 3 and 4, respectively.

The identification results of LOLIMOT and the proposed algorithm for terminal voltage and electrical power are summarized in Tabs. 1 and 2, respectively.

It can be followed from Tabs. 1 and 2 that the proposed algorithm yields a model with a more reduced number of effective parameters and better validation performance.

\section{CONCLUSIONS}

Nonlinear identification of a synchronous machine using multiple model structure is described in this paper. In the proposed identification algorithm, neither the complex global structure nor multiple model structure with a high number of local models are used. Instead, the advantages of both methods are combined together to identify a model with fewer number of effective parameters and fewer local models. The proposed method uses local polynomial (or linear) models for complex (or simple) operating regions.

To obtain a more precise model, at each iteration, a choice is made between increasing the number of optimal local models or increasing the complexity of each local model. By this method, each local model order also is determined. The proposed method has been verified by studies using actual data obtained on a physical synchronous machine and performed much better than previous algorithms with fewer parameters.

In this paper, terminal voltage and the active power are considered as the outputs of the system and the field voltage as the input of the system. Experimental results show that the proposed method can provide a more precise model with fewer parameters. The obtained model can be used for system analysis and controller design, and is planned to be used for designing a power system stabilizer (PSS) in a predictive on-line control structure.

\section{Appendix — The Orthogonal Least Squares with A-optimality algorithm}

A linear-in-parameters model can be formulated as

$$
y(k)=\sum_{i=1}^{n} \theta_{i}(k) \varphi_{i}(k)+e(k), \quad i=1,2, \ldots, N
$$

where $\varphi_{i}(k), i=1,2, \ldots, n$ are all candidate model terms, $e(k)$ is an uncorrelated model residual sequence with zero mean and variance of $\sigma^{2}$ and $\theta_{i}, i=1,2, \ldots, n$ are the unknown parameters to be estimated.

Equation (9) can be represented in matrix form as

$$
\boldsymbol{y}=U \boldsymbol{\theta}+\boldsymbol{E}
$$

where $\boldsymbol{y}=[y(1), \ldots, y(N)]^{\top}, U=\left[\boldsymbol{\varphi}_{1}, \ldots, \boldsymbol{\varphi}_{n}\right]$, $\boldsymbol{\varphi}_{i}=\left[\varphi_{i}(1), \ldots, \varphi_{i}(N)\right]^{\top}, \boldsymbol{\theta}=\left[\theta_{1}, \ldots, \theta_{n}\right]^{\top}$, $\boldsymbol{E}=[e(1), \ldots, e(N)]^{\top}$.

An orthogonal decomposition of $U$ is

$$
U=P A
$$

where $A$ is an $n \times n$ unit upper triangular matrix and $P$ is an $N \times n$ matrix with orthogonal columns $\boldsymbol{p}_{i}$ such that

$$
P^{\top} P=\operatorname{diag}\left\{p_{1}^{\top} p_{1}, p_{2}^{\top} p_{2}, \ldots, p_{n}^{\top} p_{n}\right\}
$$


so that (10) can be expressed as

$$
\boldsymbol{y}=P A \boldsymbol{\theta}+\boldsymbol{E}=P \boldsymbol{\theta}^{\circ}+\boldsymbol{E} .
$$

The orthogonal least squares solution $\boldsymbol{\theta}^{\circ}$ can be estimated from $\hat{\boldsymbol{\theta}}^{\circ}=\left(P^{\top} P\right)^{-1} P^{\top} \boldsymbol{y}$ or $\hat{\theta}_{i}^{\circ}=\frac{\boldsymbol{p}_{i}^{\top} \boldsymbol{y}}{\boldsymbol{p}_{i} \boldsymbol{p}_{\boldsymbol{i}}}$. The original parameters $\boldsymbol{\theta}$ can be estimated from

$$
A \theta=\theta^{\circ} .
$$

The mean squares error $(J)$ is computed as

$$
\begin{aligned}
J=\frac{1}{N} \boldsymbol{y}^{\top} \boldsymbol{y}-\frac{1}{N} \sum_{i=1}^{n} \theta_{i}^{\circ} \boldsymbol{p}_{i}^{\top} \boldsymbol{p}_{i}= & \frac{1}{N} \boldsymbol{y}^{\top} \boldsymbol{y}- \\
& \frac{1}{N} \sum_{i=1}^{n} \frac{\left(\boldsymbol{p}_{i}^{\top} \boldsymbol{y}\right)^{2}}{\boldsymbol{p}_{i}^{\top} \boldsymbol{p}_{i}} .
\end{aligned}
$$

To enhance model robustness, (15) is used with Aoptimality criterion. Such a criterion is defined as

$$
\min \left\{\operatorname{tr}[\operatorname{cov} \theta]=\sigma^{2} \sum_{i=1}^{n} \frac{1}{\boldsymbol{p}_{i}^{\top} \boldsymbol{p}_{i}}\right\} .
$$

Equation (15) and A-optimality are augmented as

$$
J=\frac{1}{N} \boldsymbol{y}^{\top} \boldsymbol{y}-\frac{1}{N} \sum_{i=1}^{n} \frac{\left(\boldsymbol{p}_{i}^{\top} \boldsymbol{y}\right)^{2}}{\boldsymbol{p}_{i}^{\top} \boldsymbol{p}_{i}}+\alpha \sum_{i=1}^{n} \frac{1}{\boldsymbol{p}_{i}^{\top} \boldsymbol{p}_{i}}
$$

where positive number $\alpha$ regulates the tradeoff between model approximation ability and robustness. Note that the net contribution of each term $\boldsymbol{p}_{i}$ can be computed independently as $\left(\boldsymbol{p}_{i}^{\top} \boldsymbol{y}\right)^{2} / N \boldsymbol{p}_{i}^{\top} \boldsymbol{p}_{i}+\alpha \sum_{i=1}^{n} 1 / \boldsymbol{p}_{i}^{\top} \boldsymbol{p}_{i}$.

Equation (17) can be expressed as

$$
J^{(i)}=J^{(i-1)}-\frac{1}{N} \frac{\left(\boldsymbol{p}_{i}^{\top} \mathbf{y}\right)^{2}}{\boldsymbol{p}_{i}^{\top} \boldsymbol{p}_{i}}+\alpha \frac{1}{\boldsymbol{p}_{i}^{\top} \boldsymbol{p}_{i}} .
$$

At the $i$-th iteration, a candidate term is selected as $i$-th term if it produces the smallest $J^{(i)}$ [13]. The selection procedure is terminated if $J^{\left(n^{\circ}+1\right)} \geq J^{\left(n^{\circ}\right)}$.

The identified model is expressed as

$$
y(k)=\sum_{i=1}^{n^{\circ}} \theta_{i}^{\circ}(k)+e(k), k=1, \ldots, N .
$$

The model output is represented by means of the nonorthogonal model terms

$$
\begin{aligned}
y(k)=\sum_{i=1}^{n^{\circ}} \theta_{\gamma_{i}} \varphi_{\gamma_{i}}(k) & +e(k), \\
k & =1, \ldots, N, \gamma_{i}=\{1, \ldots, n\}
\end{aligned}
$$

where the parameters $\boldsymbol{\theta}=\left[\theta_{\gamma_{1}}, \theta_{\gamma_{2}}, \ldots, \theta_{\gamma_{n}}\right]^{\top}$ can be calculated from (14). $\left\{\gamma_{1}, \gamma_{2}, \ldots, \gamma_{n^{\circ}}\right\}$ is the index set of non-zero components of $\boldsymbol{\theta}$ where $\theta_{\gamma_{i}}$ is the $\gamma_{i}$-th component in the parameter vector $\boldsymbol{\theta}$.

\section{REFERENCES}

[1] HASNI, M.-TOUHAMI, O.-IBTIOUEN, R.-FADEL, M. -CAUX, S.: J. Electrical Eng. 59 No. 2 (2008), 75-80.

[2] SOLIMAN, M.-WESTWICK, D.-MALIK, O. P.: Identification of HeffronPhillips Model Parameters for Synchronous Generators Operating in Closed Loop, IET Gener. Transm. Distrib. 2 No. 4 (2008), 530-541.

[3] MELGOZA, J.-JESUS, R.-HEYDT, G. T.-KEYHANI, A. : An Algebraic Approach for Identifying Operating Point Dependent Parameters of Synchronous Machine using Orthogonal Series Expansions, IEEE Trans. Energy Convers. 16 No. 1 (Mar 2001), 92-98.

[4] MElgOzA, J. J. R.-HEYDT, G. T.-KEYHANI, A.-AGRAWAL, B. L.-SELIN, D. : Synchronous Machine Parameter Estimation using Hartley Series, IEEE Trans. Energy Convers. 16 No. 1 (Mar 2001), 49-54.

[5] KARRARI, M.-MALIK, O. P.: Identification of HeffronPhillips Model Parameters for Synchronous Generators using Online Measurements, IEE Proc.-Gener. Transm. Distrib. 151 No. 3 (2004), 313-320.

[6] FARD, R. D.-KARRARI, M.-MALIK, O. P.: Synchronous Generator Model Identification for Control Application using Volterra Series, IEEE Trans. Energy Convers. 20 No. 4 (Dec 2005), 852-858.

[7] KARRARI, M.-MALIK, O. P.: Identification of Synchronous Generator using Adaptive Wavelet Networks, Electrical Power and Energy System, vol. 27, Elsevier, Aug 2005, pp. 113-120.

[8] SHAMSOOLLAHI, P.-MALIK, O. P. : On-Line Identification of Synchronous Generator using Neural Networks, Proceedings of the Canadian Conference on Electrical and Computer Engineering, CCECE96, Part 2, 1996, pp. 595-598.

[9] VÖRÖS, J.: Parameter Identification of Hammerstein Systems with Asymmetric Dead-Zone, J. Electrical Eng. 55 No. 1-2 (2004), 46-49.

[10] VÖRÖS, J.: An Iterative Method for Hammerstein-Wiener Systems Parameter Identification J. Electrical Eng..

[11] WEI, H. L.-BILLINGS, S. A.-ZHAO, Y.-GUO, L.: Lattice Dynamical Wavelet Neural Networks Implemented Using Particle Swarm Optimization for SpatioTemporal System Identification, IEEE Transaction on Neural Networks 20 No. 1 (Jan 2009).

[12] BAI, E. W.: Non-Parametric Nonlinear System Identification: A Data-Driven Orthogonal Basis Function Approach, IEEE Transaction on automatic control 53 No. 11 (Dec 2008).

[13] HONG, X.-HARRIS, C. J.: Nonlinear Model Structure Detection using Optimum Experimental Design and Orthogonal Least Squares, IEEE Transactions on Neural Networks 12 No. 2 (2001), 435-439.

[14] MURRAY-SMITH, R. :JOHANSEN, T. A.: Multiple Model Approaches to Modeling and Control, Taylor and Francis, London, 1997.

[15] BROWN, M. D.-FLYNN, D.-IRWIN, G. W.: Multiple Model Nonlinear Control of Synchronous Generators, Transactions of the Institute of Measurement and Control 24 (2002), 215-230.

[16] NELLES, O.: Nonlinear System Identification: From Classical Approaches to Neural Networks and Fuzzy Models, Springer Verlag, 2000.

[17] KUNDUR, P.: Power System Stability and Control, McGraw-Hill Inc., 1994.

Received 20 January 2011

Seyed Salman Ahmadi and Mehdi Karrari Biographies not supplied. 